\title{
Trend of multidrug and fluoroquinolone resistance in Mycobacterium tuberculosis isolates from 2010 to 2014 in Korea: a multicenter study
}

Hyeonseok Kim ${ }^{1}$, Jeong Ha Mok², Bohyoung Kang ${ }^{3}$, Taehoon Lee ${ }^{4}$, Hyun-Kyung Lee ${ }^{5}$, Hang Jea Jang ${ }^{6}$, $\mathrm{Yu} \mathrm{Ji} \mathrm{Cho}^{7}$, and Doosoo Jeon ${ }^{1}$

\begin{abstract}
${ }^{1}$ Department of Internal Medicine, Pusan National University Yangsan Hospital, Yangsan; ${ }^{2}$ Department of Internal Medicine, Pusan National University Hospital, Busan;

${ }^{3}$ Department of Internal Medicine, Dong-A University Hospital, Busan; ${ }^{4}$ Department of Internal Medicine, Ulsan University Hospital, Ulsan; ${ }^{5}$ Department of Internal Medicine, Inje University Busan Paik Hospital, Busan; ${ }^{6}$ Department of Internal Medicine, Inje University Haeundae Paik Hospital, Busan; ${ }^{7}$ Department of Internal Medicine, Gyeongsang National University Hospital, Jinju, Korea
\end{abstract}

Received: February 6, 2018

Revised : March 2, 2018

Accepted: March 14, 2018

\section{Correspondence to}

Doosoo Jeon, M.D.

Department of Internal Medi-

cine, Pusan National University

Yangsan Hospital, 20 Geumo-ro,

Mulgeum-eup, Yangsan 50612,

Korea

Tel: $+82-55-360-1414$

Fax: +82-55-360-1759

E-mail: sooliıo@hanmail.net
Background/Aims: This study was conducted to evaluate the recent prevalence and trend of anti-tuberculosis (TB) drug resistance with a focus on multidrug-resistance (MDR) and fluoroquinolone resistance in South Korea.

Methods: We retrospectively reviewed the drug susceptibility testing results of culture-confirmed Mycobacterium tuberculosis isolates collected from 2010 to 2014 at seven tertiary hospitals in South Korea.

Results: A total of 5,599 cases were included: 4,927 (88.0\%) were new cases and 672 (12.0\%) were previously treated cases. The MDR rate has significantly decreased from $6.0 \%$ in 2010 to $3.0 \%$ in 2014 among new cases, and from $28.6 \%$ in 2010 to $18.4 \%$ in 2014 among previously treated cases $(p<0.001$ and $p=0.027$, respectively). The resistance rate to any fluoroquinolone was $0.8 \%(43 / 5,221)$ in non-MDR-TB patients, as compared to $26.2 \%$ (99/378) in MDR-TB patients $(p<0.001)$. There was no significant change in the trend of fluoroquinolone resistance among both nonMDR-TB and MDR-TB patients. Among the 43 non-MDR-TB patients with fluoroquinolone resistance, 38 (88.4\%) had fluoroquinolone mono-resistant isolates.

Conclusions: The prevalence of MDR-TB has significantly decreased from 2010 to 2014. The prevalence of fluoroquinolone resistance among non-MDR-TB patients was low, but the existence of fluoroquinolone mono-resistant TB may be a warning on the widespread use of fluoroquinolone in the community.

Keywords: Drug resistance; Tuberculosis; Tuberculosis, multidrug-resistant; Fluoroquinolones; Korea

\section{INTRODUCTION}

Drug-resistant tuberculosis (TB) is developed and subsequently amplified under poor TB control programs, such as inadequate treatment, patient care, and infection control [1]. Therefore, data on the prevalence and trends of anti-TB drug resistance can provide key in- formation on understanding TB situation as well as determining the effectiveness of TB control programs in a country.

In South Korea, nationwide drug surveillance had been conducted on four occasions between 1994 and 2004, which was a representative sample survey of patients in public health centers [2]. These surveys showed 
that multidrug-resistance (MDR) rate in new cases had been gradually increasing from $1.6 \%$ in 1994 to $2.2 \%$ in $1999,2.4 \%$ in 2003 , and $2.7 \%$ in 2004 , respectively [2]. Thereafter, the nationwide trend of anti-TB drug resistance could not be estimated because this survey was stopped and replaced with a new TB notification system. In the 2000s, attempts to estimate the number and trend of MDR-TB patients were made by collecting the data from seven institutions that performed most drug susceptibility testing (DST) in Korea [3,4]. Despite the progress in TB control since the 2010s, there has been little data demonstrating how these changes have influenced the trend of drug resistance in South Korea.

Fluoroquinolones (FQs) are the backbone of MDRTB treatment regimens and FQ resistance is associated with poor treatment outcomes [5-7]. Recently, a survey on FQ resistance has been highlighted because it is crucial to determine the feasibility of introducing a standardized shorter MDR-TB regimen in a country $[8,9]$. Globally, the data on the prevalence of FQ resistance at the population level are limited, because testing for FQ resistance is not routinely performed in most TB endemic areas. In contrast, universal DST has been recommended to all TB patients from the 2005 Korean guidelines [10]. DST examines 15 tested drugs including three FQs (ofloxacin, levofloxacin, and moxifloxacin). However, there have been little data on the prevalence and trend of FQ resistance in South Korea.

We recently reported high resistance rate to additional drugs among MDR-TB patients in seven university hospitals in South Korea [11]. We performed additional analysis in the same cohort to evaluate the recent prevalence and trend of anti-TB drug resistance focusing on MDR and FQ resistance.

\section{METHODS}

\section{Study design and data collection}

We included patients who were diagnosed with culture-confirmed TB and had results of DST between January 2010 and December 2014, at seven university-affiliated tertiary hospitals in Busan, Ulsan, and Gyeongsangnam-do in South Korea. We retrospectively reviewed the medical records of those patients, and the following data were collected: age, sex, history of previ- ous TB treatment, type of specimen, and results of phenotypic DST. Previous TB treatment history was assessed by physicians through patient history taking. We excluded patients who had an unknown history of previous TB treatment and those who had duplicated in-hospital or inter-hospital records. If a patient had more than one DST result, the earlier result was selected. If the patient had DST results for both pulmonary and extra-pulmonary specimens, results for the pulmonary specimen were selected.

\section{Drug susceptibility test}

DST was performed on Mycobacterium tuberculosis (MTB) isolates, collected prior to treatment initiation or regimen change. For DST, six hospitals sent MTB isolates to the Korean Institute of Tuberculosis, and one hospital sent them to the Green Cross Reference Laboratory. The protocol used and critical concentration references for resistance were the same in both laboratories. The drug susceptibility was determined by the absolute concentration method using the Lowenstein-Jensen medium. The drugs and their critical concentrations for resistance were as follows: isoniazid $0.2 \mu \mathrm{g} / \mathrm{mL}$, rifampin $40 \mu \mathrm{g} / \mathrm{mL}$, ethambutol $2.0 \mu \mathrm{g} / \mathrm{mL}$, rifabutin $20 \mu \mathrm{g} / \mathrm{mL}$, streptomycin $10 \mu \mathrm{g} / \mathrm{mL}$, amikacin $40 \mu \mathrm{g} / \mathrm{mL}$, kanamycin $40 \mu \mathrm{g} / \mathrm{mL}$, capreomycin $40 \mu \mathrm{g} / \mathrm{mL}$, ofloxacin $2.0 \mu \mathrm{g} / \mathrm{mL}$, levofloxacin $2.0 \mu \mathrm{g} / \mathrm{mL}$, moxifloxacin $2.0 \mu \mathrm{g} / \mathrm{mL}$, prothionamide 40 $\mu \mathrm{g} / \mathrm{mL}$, cycloserine $30 \mu \mathrm{g} / \mathrm{mL}$, and para-aminosalicylic acid $1.0 \mu \mathrm{g} / \mathrm{mL}$. Pyrazinamide susceptibility was determined using a pyrazinamidase test.

\section{Definitions}

Patients were classified based on their history of previous TB treatment. New patients were defined as those who had never been treated for TB or who had received treatment for $<1$ month. Previously treated patients were defined as those who had received $\geq 1$ month of anti-TB drugs in the past and were further classified according to the World Health Organization (WHO)'s definition as follows [12]: relapse patients were those who were declared cured or those who completed the treatment, and were diagnosed with a recurrent episode of TB; treatment after failure patients were those whose treatment failed and treatment after loss to follow-up patients were those who were declared lost to follow-up at the end of their most recent course of treatment. 
Drug-resistant cases were classified based on the DST results as follows: any drug resistance was defined as resistance to any anti-TB drugs; MDR, as resistance to at least both isoniazid and rifampicin; extensive-drug resistance (XDR), as resistance to any $\mathrm{FQ}$, and at least one of the three second-line injectable drugs (kanamycin, amikacin, and capreomycin) in addition to MDR; pre-XDR TB, as resistance to either a FQ or second line-injectable drugs but not both, in addition to MDR; and FQ mono-resistance, as resistance to any FQ but not to other drugs.

\section{Statistical analysis}

Data were presented as mean \pm standard deviations for continuous variables and as numbers (with percentages) for categorical variables. Continuous variables were compared using the independent samples $t$ test, and categorical variables were compared using the Pearson chi-square test or Fisher exact test, respectively. To evaluate the annual drug resistance trend, chi-square test for trend was performed. All tests were two-tailed, and $p<0.05$ indicated statistical significance. Statistical analysis was performed using SPSS 17.0 version (SPSS Inc., Chicago, IL, USA).

\section{Ethics statement}

The present study protocol was reviewed and approved by the Institutional Review Board of Pusan National University Hospital (IRB approval number: E-2015117), and the requirement for obtaining informed consent was waived.

\section{RESULTS}

\section{Patients' characteristics}

A total of 5,607 patients who were diagnosed with culture-confirmed TB and had DST results were screened. Among them, eight patients were excluded; four had unknown history of previous TB treatment and four were duplicated between hospitals. As a result, 5,599 patients were finally included. The demographics and characteristics of the total patients are shown in Table 1.

\section{Trend in drug resistance rate}

Among 4,927 new cases, the drug resistance pattern and trends from 2010 to 2014 are shown in Table 2 . The resistance rate to any drug and isoniazid was $13.5 \%$ and $10.8 \%$, respectively. MDR, pre-XDR, and XDR rates

Table 1. Demographics and baseline characteristics

\begin{tabular}{|c|c|c|c|c|}
\hline Characteristic & Male & Female & Total & $p$ value \\
\hline No. of cases & $3,266(58.3)$ & $2,333(41.7)$ & $5,599(100)$ & \\
\hline Age, yr & $53.0 \pm 21.4$ & $53.6 \pm 20.9$ & $53.2 \pm 21.2$ & 0.351 \\
\hline Age group, yr & & & & $<0.001$ \\
\hline $0-19$ & $100(1.8)$ & $57(1.0)$ & $157(2.8)$ & \\
\hline $20-39$ & $685(12.2)$ & $702(12.6)$ & $1,387(24.8)$ & \\
\hline $40-59$ & $1,246(22.3)$ & $540(9.6)$ & $1,786(31.9)$ & \\
\hline$\geq 60$ & $1,235(22.1)$ & $1,034(18.4)$ & $2,269(40.5)$ & \\
\hline Specimen & & & & $<0.001$ \\
\hline Pulmonary & $3,111(55 \cdot 6)$ & $2,168(38.7)$ & $5,279(94 \cdot 3)$ & \\
\hline Extra-pulmonary & $155(2.8)$ & $165(2.9)$ & $320(5 \cdot 7)$ & \\
\hline Case classification & & & & $<0.001$ \\
\hline New & $2,788(49.8)$ & $2,139(38.2)$ & $4,927(88.0)$ & \\
\hline Previously treated & $478(8.5)$ & $194(3 \cdot 5)$ & $672(12.0)$ & \\
\hline Relapse & $404(7.2)$ & $162(2.9)$ & $566(10.1)$ & \\
\hline Failure & $23(0.4)$ & $12(0.2)$ & $35(0.6)$ & \\
\hline Loss to follow-up & $51(0.9)$ & $20(0.4)$ & $71(1.3)$ & \\
\hline
\end{tabular}

Values are presented as number (\%) or mean \pm SD. 
Table 2. Trend in the anti-tuberculosis drug resistance rate of Mycobacterium tuberculosis isolates from new cases

\begin{tabular}{|c|c|c|c|c|c|c|c|}
\hline \multirow{2}{*}{ Variable } & \multicolumn{5}{|c|}{ Year } & \multirow{2}{*}{ Total } & \multirow{2}{*}{$p$ for trend } \\
\hline & 2010 & 2011 & 2012 & 2013 & 2014 & & \\
\hline No. of cases & 836 & 956 & 1,043 & 1,089 & 1,003 & 4,927 & \\
\hline Isoniazid & $110(13.2)$ & $89(9.3)$ & $111(10.6)$ & $124(11.4)$ & $97(9.7)$ & $531(10.8)$ & 0.172 \\
\hline Rifampin & $53(6.3)$ & $61(6.4)$ & $48(4 \cdot 6)$ & $47(4 \cdot 3)$ & $35(3 \cdot 5)$ & $244(5 \cdot 0)$ & $<0.001$ \\
\hline Rifabutin & $36(4 \cdot 3)$ & $44(4.6)$ & $36(3.5)$ & $33(3.0)$ & $24(2.4)$ & $173(3.5)$ & 0.004 \\
\hline Ethambutol & $34(4.1)$ & $43(4 \cdot 5)$ & $34(3 \cdot 3)$ & $35(3.2)$ & $28(2.8)$ & $174(3 \cdot 5)$ & 0.044 \\
\hline Pyrazinamide & $16(1.9)$ & $26(2.7)$ & $9(0.9)$ & $16(1.5)$ & $16(1.6)$ & $83(1.7)$ & 0.160 \\
\hline Streptomycin & $26(3.1)$ & $37(3.9)$ & $45(4 \cdot 3)$ & $44(4 \cdot 0)$ & $42(4.2)$ & $194(3.9)$ & 0.276 \\
\hline Kanamycin & $7(0.8)$ & $7(0.7)$ & $10(1.0)$ & $9(0.8)$ & $7(0.7)$ & $40(0.8)$ & 0.863 \\
\hline Capreomycin & $7(0.8)$ & $6(0.6)$ & $6(0.6)$ & $9(0.8)$ & $6(0.6)$ & $34(0.7)$ & 0.804 \\
\hline Amikacin & $7(0.8)$ & $6(0.6)$ & $8(0.8)$ & $7(0.6)$ & $7(0.7)$ & $35(0.7)$ & 0.806 \\
\hline Ofloxacin & $16(1.9)$ & $16(1.7)$ & $19(1.8)$ & $14(1.3)$ & $11(1.1)$ & $76(1.5)$ & 0.111 \\
\hline Levofloxacin & $15(1.8)$ & $14(1.5)$ & $18(1.7)$ & $14(1.3)$ & $8(0.8)$ & $69(1.4)$ & 0.071 \\
\hline Moxifloxacin & $11(1.3)$ & $11(1.2)$ & $15(1.4)$ & $12(1.1)$ & $7(0.7)$ & $56(1.1)$ & 0.243 \\
\hline Prothionamide & $13(1.6)$ & $14(1.5)$ & $10(1.0)$ & $10(0.9)$ & $5(0.5)$ & $52(1.1)$ & 0.013 \\
\hline Cycloserin & $2(0.2)$ & $4(0.4)$ & $2(0.2)$ & $1(0.0)$ & $3(0.3)$ & $12(0.2)$ & 0.679 \\
\hline Para-aminosalicylic acid & $25(3.0)$ & $30(3.1)$ & $27(2.6)$ & $27(2.5)$ & $18(1.8)$ & $127(2.6)$ & 0.059 \\
\hline Any drug & $129(15.4)$ & $116(12.1)$ & $147(14.1)$ & $147(13 \cdot 5)$ & $127(12.7)$ & $666(13.5)$ & 0.276 \\
\hline MDR & $50(6.0)$ & $54(5.6)$ & $41(3.9)$ & $41(3.8)$ & $30(3.0)$ & $216(4 \cdot 4)$ & $<0.001$ \\
\hline Pre-XDR & $6(0.7)$ & $9(0.9)$ & $7(0.7)$ & $8(0.7)$ & $7(0.7)$ & $37(0.8)$ & 0.766 \\
\hline XDR & $5(0.6)$ & $5(0.5)$ & $4(0.4)$ & $5(0.5)$ & $1(0.1)$ & $20(0.4)$ & 0.123 \\
\hline
\end{tabular}

Values are presented as number (\%).

MDR, multidrug resistance; XDR, extensively drug resistance.

were $4.4 \%, 0.8 \%$, and $0.4 \%$, respectively. The MDR rate among new cases has significantly decreased from $6.0 \%$ in 2010 to $5.6 \%$ in $2011,3.9 \%$ in $2012,3.8 \%$ in 2013 , and $3.0 \%$ in $2014(p<0.001)$. The annual resistance rate to any drug decreased from $15.4 \%$ in 2010 to $12.7 \%$ in 2014 , but not statistically significant $(p=0.276)$. The annual resistance rate to rifampicin, rifabutin, ethambutol, and prothionamide showed a significant decreasing trend, while other drugs including three FQs did not.

The drug resistance pattern and trends of previously treated cases are shown in Table 3. The resistance rates to any drug and isoniazid were $37.6 \%$ and $35.4 \%$, respectively. MDR, pre-XDR, and XDR rates were $24.1 \%, 6.1 \%$, and $4.0 \%$, respectively. The MDR rate has significantly decreased from $28.6 \%$ in 2010 to $18.4 \%$ in 2014 ( $p=0.027$ ). The drug resistance rate to rifampicin, capreomycin, cycloserine, and PAS showed a significant decreasing trend, while other drugs including three FQs did not.

\section{Trend in fluoroquinolone resistance}

The resistance rate to any FQ was $0.8 \%(43 / 5,221)$ in nonMDR-TB patients, and 26.2\% (99/378) in MDR-TB patients $(p<0.001)$. The trend of FQ resistance did not significantly change in both non-MDR-TB and MDRTB patients (Table 4), which was consistent across the seven hospitals.

Among the 43 non-MDR-TB patients with FQ resistance, 38 (88.4\%) had isolates with FQ mono-resistance and five (11.6\%) had combined resistance. Drug resistance pattern of these patients are shown in Table 5. Of the 43 patients, 24 (44\%) were women with a mean of 60 years. Thirty-seven patients (86.0\%) were new cases and six (14.0\%) were previously treated cases receiving firstline agents only. None of them had previously received FQ for TB treatment. FQ mono-resistant TB was found in all seven hospitals throughout the study periods. 
Table 3. Trend in the anti-tuberculosis drug resistance rate of Mycobacterium tuberculosis isolates from previously treated cases

\begin{tabular}{|c|c|c|c|c|c|c|c|}
\hline \multirow{2}{*}{ Variable } & \multicolumn{5}{|c|}{ Year } & \multirow{2}{*}{ Total } & \multirow{2}{*}{$p$ for trend } \\
\hline & 2010 & 2011 & 2012 & 2013 & 2014 & & \\
\hline No. of cases & 126 & 152 & 131 & 138 & 125 & 672 & \\
\hline Isoniazid & $48(38.1)$ & $54(35 \cdot 5)$ & $51(38.9)$ & $47(34.1)$ & $38(30.4)$ & $238(35.4)$ & 0.223 \\
\hline Rifampin & $37(29.4)$ & $47(30.9)$ & $26(19.8)$ & $35(25 \cdot 4)$ & $24(19.2)$ & $169(25.1)$ & 0.032 \\
\hline Rifabutin & $23(18.3)$ & $35(23.0)$ & $20(15 \cdot 3)$ & $27(19.6)$ & $19(15.2)$ & $124(18.5)$ & 0.352 \\
\hline Ethambutol & $24(19.0)$ & $25(16.4)$ & $14(10.7)$ & $23(16.7)$ & $19(15 \cdot 2)$ & $105(15 \cdot 6)$ & 0.491 \\
\hline Pyrazinamide & $15(11.9)$ & 15 (9.9) & $8(6.1)$ & $23(16.7)$ & $10(8.0)$ & $71(10.6)$ & 1.000 \\
\hline Streptomycin & $12(9.5)$ & $11(7.2)$ & 13 (9.9) & $12(8.7)$ & $11(8.8)$ & $59(8.8)$ & 1.000 \\
\hline Kanamycin & $11(8.7)$ & $9(5 \cdot 9)$ & $1(0.8)$ & $11(8.0)$ & $2(1.6)$ & $34(5.1)$ & 0.057 \\
\hline Capreomycin & $11(8.7)$ & $4(2.6)$ & $1(0.8)$ & $9(6.5)$ & $1(0.8)$ & $26(3.9)$ & 0.043 \\
\hline Amikacin & $11(8.7)$ & $3(2.0)$ & $1(0.8)$ & $9(6.5)$ & $2(1.6)$ & $26(3.9)$ & 0.113 \\
\hline Ofloxacin & $15(11.9)$ & $16(10.5)$ & $8(6.1)$ & $17(12.3)$ & $7(5.6)$ & $63(9.4)$ & 0.216 \\
\hline Levofloxacin & $14(11.1)$ & $13(8.6)$ & $8(6.1)$ & $15(10.9)$ & $7(5 \cdot 6)$ & $57(8.5)$ & 0.296 \\
\hline Moxifloxacin & $12(9.5)$ & $12(7 \cdot 9)$ & $5(3.8)$ & $13(9.4)$ & $7(5 \cdot 6)$ & $49(7 \cdot 3)$ & 0.424 \\
\hline Prothionamide & $10(7 \cdot 9)$ & $12(7 \cdot 9)$ & $4(3.1)$ & $11(8.0)$ & $4(3.2)$ & $41(6.1)$ & 0.165 \\
\hline Cycloserin & $7(5.6)$ & $6(3.9)$ & $1(0.8)$ & $2(1.4)$ & $1(0.8)$ & $17(2.5)$ & 0.005 \\
\hline Para-aminosalicylic acid & $12(9 \cdot 5)$ & $20(13.2)$ & $9(6.9)$ & $13(9.4)$ & $4(3.2)$ & $58(8.6)$ & 0.033 \\
\hline Any drug & $50(8.7)$ & $56(36.8)$ & $54(41.2)$ & $51(37.0)$ & $42(33.6)$ & $253(37.6)$ & 0.406 \\
\hline MDR & $36(28.6)$ & $45(29.6)$ & $25(19.1)$ & $33(23.9)$ & $23(18.4)$ & $162(24.1)$ & 0.027 \\
\hline Pre-XDR & $6(4.8)$ & $15(9.9)$ & $7(5 \cdot 3)$ & $8(5.8)$ & $5(4.0)$ & $41(6.1)$ & 0.355 \\
\hline XDR & $10(7 \cdot 9)$ & $6(3.9)$ & $0(0.0)$ & $9(6.5)$ & $2(1.6)$ & $27(4.0)$ & 0.089 \\
\hline
\end{tabular}

Values are presented as number (\%).

MDR, multidrug resistance; XDR, extensively drug resistance.

Table 4. Trend in the fluoroquinolone resistance rate of Mycobacterium tuberculosis isolates from patients with non-MDR-TB and MDR-TB

\begin{tabular}{|c|c|c|c|c|c|c|c|}
\hline \multirow{2}{*}{ Variable } & \multicolumn{5}{|c|}{ Year } & \multirow{2}{*}{ Total } & \multirow{2}{*}{$p$ for trend } \\
\hline & 2010 & 2011 & 2012 & 2013 & 2014 & & \\
\hline Non-MDR & 876 & 1,009 & 1,108 & 1,153 & 1,075 & 5,221 & \\
\hline Any FQ & $9(1.0)$ & $7(0.7)$ & $12(1.1)$ & $6(0.5)$ & $9(0.8)$ & $43(0.8)$ & 0.544 \\
\hline Ofloxacin & $9(1.0)$ & $7(0.7)$ & $12(1.1)$ & $6(0.5)$ & $9(0.8)$ & $43(0.8)$ & 0.544 \\
\hline Levofloxacin & $9(1.0)$ & $5(0.5)$ & $12(1.1)$ & $6(0.5)$ & $6(0.6)$ & $38(0.7)$ & 0.291 \\
\hline Moxifloxacin & $6(0.7)$ & $4(0.4)$ & $9(0.8)$ & $5(0.4)$ & $5(0.5)$ & $29(0.6)$ & 0.588 \\
\hline MDR & 86 & 99 & 66 & 74 & 53 & 378 & \\
\hline Any FQ & $22(25.6)$ & $28(28.3)$ & $15(22.7)$ & $25(33.8)$ & $9(17.0)$ & $99(26.2)$ & 0.659 \\
\hline Ofloxacin & $22(25.6)$ & $25(25 \cdot 3)$ & $15(22.7)$ & $25(33.8)$ & $9(17 \cdot 0)$ & $96(25 \cdot 4)$ & 0.803 \\
\hline Levofloxacin & $20(23 \cdot 3)$ & $22(22.2)$ & $14(21.2)$ & $23(31.1)$ & $9(17 \cdot 0)$ & $88(23 \cdot 3)$ & 0.987 \\
\hline Moxifloxacin & $17(19.8)$ & $19(19.2)$ & $11(16.7)$ & $20(27.0)$ & $9(17.0)$ & $76(20.1)$ & 0.757 \\
\hline
\end{tabular}

Values are presented as number (\%).

MDR, multidrug-resistance; TB, tuberculosis; FQ, fluoroquinolone. 
Table 5. Drug susceptibility pattern of Mycobacterium tuberculosis isolates from 43 non-MDR-TB patients with fluoroquinolone resistance

\begin{tabular}{|c|c|c|c|c|}
\hline & Ofloxacin & Levofloxacin & Moxifloxacin & No. of cases \\
\hline \multicolumn{5}{|l|}{ Mono-resistance $(\mathrm{n}=38)$} \\
\hline & $\mathrm{R}$ & $\mathrm{R}$ & $\mathrm{R}$ & 25 \\
\hline & $\mathrm{R}$ & $\mathrm{S}$ & S & 2 \\
\hline & $\mathrm{R}$ & $\mathrm{R}$ & S & 1 \\
\hline & $\mathrm{R}$ & $\mathrm{S}$ & $\mathrm{R}$ & 10 \\
\hline \multicolumn{5}{|l|}{ Combined resistance $(\mathrm{n}=5)$} \\
\hline \multicolumn{4}{|c|}{ Isoniazid, ethambutol, streptomycin, prothionamide } & 1 \\
\hline \multicolumn{4}{|c|}{ Isoniazid, ethambutol, kanamycin, cycloserine, PAS } & 1 \\
\hline & \multicolumn{3}{|c|}{ Isoniazid, streptomycin, PAS } & 1 \\
\hline & \multicolumn{3}{|c|}{ Isoniazid, streptomycin } & 1 \\
\hline & \multicolumn{3}{|c|}{ Isoniazid, prothionamide } & 1 \\
\hline
\end{tabular}

MDR, multidrug-resistance; TB, tuberculosis; R, resistant; S, susceptible; PAS, para-aminosalicylic acid.

\section{DISCUSSION}

Our results demonstrated both positive and negative findings regarding the current TB situation in South Korea. On the positive side, the MDR rate has gradually decreased among both new and previously treated patients. Conversely, the FQ resistance rate is $26.2 \%$ and the trend of FQ resistance remains constant among MDR-TB patients. Although the prevalence of FQ resistance among non-MDR-TB patients is less than $1 \%$, $88.4 \%$ of them had FQ mono-resistant isolates.

The result of our study, demonstrating the decreasing trend in MDR rate, is consistent with the trend in the number of notified MDR-TB cases in South Korea [13]. These number peaked at 1,212 in 2012, but gradually decreased to 951 in 2013,858 in 2014,787 in 2015 , and 852 in 2016. The decreasing trend in MDR rate observed in our cohort may reflect the positive effects of current TB control program in South Korea. During the last decade, TB control has been progressively improving in South Korea. The diagnosis of TB and drug-resistant TB has become faster and more accurate with the introduction of liquid culture medium and rapid molecular DST, leading to immediate and effective treatment. Treatment outcomes of MDR-TB patients have improved. A large retrospective multicenter study ( $\mathrm{n}=$ 1,407 MDR-TB patients) published in 2008 showed poor outcomes; a treatment success rate of $46.2 \%$ in
MDR-TB and $29.3 \%$ in XDR-TB patients [14]. Since then, two retrospective cohort studies published in 2015 suggested that treatment outcomes were progressively improving, largely due to the use of later generation FQs and linezolid $[15,16]$. In 2016, a prospective multicenter study ( $\mathrm{n}=151 \mathrm{MDR}-\mathrm{TB}$ patients) reported an $82.1 \%$ of treatment success rate in patients with FQ-susceptible MDR-TB [17]. Public-private mix may have contributed to the reduction of MDR-TB through better patient care and gradually improved on the accuracy of TB notification on previous treatment history.

In our study, majority of patients with rifampicin-resistance revealed MDR-TB patients. Among the 413 patients with rifampin-resistant isolates, 88.5\% (216/244) of new patients and $95.9 \%(162 / 169)$ of previously treated patients were also resistant to isoniazid. This result suggests that when rifampicin resistance is detected using the Xpert test, the patient should be treated with MDR-TB regimen in South Korea until the phenotypic DST results are available.

Despite the decreasing trend in MDR-TB, the prevalence of FQ resistance among MDR-TB patients was $26.2 \%$ in our cohort, which was higher than $21 \%$ of global estimate on FQ resistance [8], $11 \%$ in Australia [18], and $17.6 \%$ in Europe [19]. In a population-based survey coordinated by the WHO, ofloxacin resistance rate among rifampicin-resistant $\mathrm{TB}$ patients varied: 25.0\% in Azerbaijan, $16.0 \%$ in Bangladesh, $30.7 \%$ in Be- 
larus, $21.8 \%$ in Pakistan, and $18.3 \%$ in South Africa [9]. Considering the high rate of additional drug resistance among MDR-TB patients, the effective treatment of MDR-TB still pose a challenge in South Korea [11]. Furthermore, the trend of FQ resistance did not change among MDR-TB patients, indicating a need for more comprehensive TB control program. A study in Taiwan showed that FQ resistance rates significantly decreased following the implementation of a successful TB control program [20].

There were a few reports on the prevalence and trend of FQ resistance in non-MDR-TB patients in South Korea. In a nationwide survey, ofloxacin resistance rate among new cases was $0.04 \%$ in 1994, which had gradually increased to $0.8 \%$ in 2004 [2]. In a retrospective study conducted in a single hospital from 1997 to 2005, ofloxacin resistance rate was 30.3\% (83/274) in MDR-TB patients and $0.4 \%(11 / 2,514)$ in non-MDR-TB patients, and there was no significant change in the trend of FQ resistance [21]. In a population-based study coordinated by the WHO, ofloxacin resistance rate in non-MDR-TB patients was variable in five countries; $0.7 \%$ in Azerbaijan, $4.6 \%$ in Bangladesh, $2.9 \%$ in Belarus, $11.1 \%$ in Pakistan, and $0.4 \%$ in South Africa [9]. FQ resistance in nonMDR-TB patients was $0.8 \%$ in our cohort, which is an indicator that could be compared to the recent prevalence of FQ resistance in TB endemic countries.

A major reason for the development FQ resistance in MDR-TB patients is suboptimal treatment with FQ-containing regimen $[18,22]$. In a recently published Korean study, ofloxacin resistance rate among MDR-TB patients was significantly different between the public and private sector [23]. This finding suggested that resistance to second-line drugs including FQs were amplified during MDR-TB treatment. However, the mechanism of generating FQ resistance in non-MDR-TB patients remains unclear. In our study, $88.4 \%$ of non-MDR-TB patients with FQ resistance had FQ mono-resistant isolates. None of them had previously been treated with a FQ for TB. There was no evidence of an outbreak of TB strain with FQ mono-resistance, since these patients were sporadically distributed in all hospitals and throughout the study periods. Therefore, these patients were likely to have acquired FQ resistance from exposure to FQ prior to TB diagnosis. FQ exposure prior to T'B diagnosis has been associated with FQ resistance, particularly when FQ exposure occurs $>60$ days before TB diagnosis and for longer than 10 days [24-26]. FQ mono-resistant TB has been reported in several countries [27,28]. Therefore, the concern has been raised that FQ-resistant TB may be generated and amplified due to the widespread use of FQs in weak healthcare systems [29]. Because of the limitations of a retrospective study, we could not determine the association between FQ use and FQ resistance in each patient. However, the existence of FQ mono-resistant TB should be regarded as a new warning against the widespread use of FQ in the community.

This study had several limitations. First, previous TB treatment history might have been misclassified in some patients because of a retrospective study conducted in private hospitals. We did not assess the patient's treatment history in connection with the Korean Tuberculosis Surveillance System. Second, our results are not representative of the general Korean population. Our study was conducted in private tertiary hospitals in some areas; thus, might be biased. However, the notified TB cases of the region belong to the seven hospitals were $16.3 \%$ of total notified TB cases in 2014 [30]. In addition, $91.4 \%$ of total TB cases were notified from the private sector, and $86.3 \%$ of them were notified from tertiary hospitals [30]. Considering this situation, our result may closely reflect the overall TB situation in South Korea. Further studies based on a representative sample of the TB population are warranted.

In conclusion, this study showed a decreasing trend in MDR, but not in FQ resistance. Although the prevalence of FQ resistance among non-MDR-TB patients was less than $1 \%$, the existence of FQ mono-resistant TB may be a warning against the widespread use of FQ in the community.

\section{KEY MESSAGE}

1. The multidrug-resistance (MDR) rate has gradually decreased from 2010 to 2014 among both new and previously treated patients.

2. The prevalence of fluoroquinolone (FQ) resistance among non-MDR-tuberculosis patients was less than $1 \%$, but $88.4 \%$ of them had FQ mono-resistant isolates. 


\section{Conflict of interest}

No potential conflict of interest relevant to this article was reported.

\section{REFERENCES}

1. Dheda K, Gumbo T, Maartens G, et al. The epidemiology, pathogenesis, transmission, diagnosis, and management of multidrug-resistant, extensively drug-resistant, and incurable tuberculosis. Lancet Respir Med 2017;5:291-360.

2. Bai GH, Park YK, Choi YW, et al. Trend of anti-tuberculosis drug resistance in Korea, 1994-2004. Int J Tuberc Lung Dis 2007;11:571-576.

3. Kim BJ, Lee IH, Lee DH, et al. The current status of multidrug-resistant tuberculosis in Korea. Tuberc Respir Dis 2006;60:404-411.

4. Kim SY, Kim HJ, Kim CK, et al. The recent status of multidrug- and extensively drug-resistant tuberculosis in Korea. Tuberc Respir Dis 2010;68:146-154.

5. Johnston JC, Shahidi NC, Sadatsafavi M, Fitzgerald JM. Treatment outcomes of multidrug-resistant tuberculosis: a systematic review and meta-analysis. PLoS One 2009;4:e6914.

6. Ahuja SD, Ashkin D, Avendano M, et al. Multidrug resistant pulmonary tuberculosis treatment regimens and patient outcomes: an individual patient data meta-analysis of 9,153 patients. PLoS Med 2012;9:e1001300.

7. Falzon D, Gandhi N, Migliori GB, et al. Resistance to fluoroquinolones and second-line injectable drugs: impact on multidrug-resistant TB outcomes. Eur Respir J 2013;42:156-168.

8. World Health Organization. Global tuberculosis report 2016 [Internet]. Geneva: World Health Organization, c2018 [cited 2018 Jul 3]. Available from: http://apps.who. int/iris/bitstream/handle/10665/250441/9789241565394-e ng.pdf;jsessionid=A7DBC85BFAE4B2BCCA2727EA99FB6AFE?sequence $=1$.

9. Zignol M, Dean AS, Alikhanova N, et al. Population-based resistance of Mycobacterium tuberculosis isolates to pyrazinamide and fluoroquinolones: results from a multicountry surveillance project. Lancet Infect Dis 2016;16:1185-1192.

10. The Korean Academy of Tuberculosis and Respiratory Disease. Korean Guidelines for Tuberculosis, Fourth Edition [Internet]. Seoul (KR): The Korean Academy of
Tuberculosis and Respiratory Disease, 2005 [cited 2018 Jul 3]. Available from: http://www.lungkorea.org/bbs/index. html? code=guide\&page $=3$.

11. Mok JH, Kang BH, Lee T, et al. Additional drug resistance patterns among multidrug-resistant tuberculosis patients in Korea: implications for regimen design. J Korean Med Sci 2017;32:636-641.

12. World Health Organization. Definitions and reporting framework for tuberculosis: 2013 revision [Internet]. Geneva: World Health Organization, c2018 [cited 2018 Jul 3]. Available from: http://www.who.int/tb/publications/definitions/en.

13. Korea Centers for Disease Control and Prevention. Annual report on the notified tuberculosis patients in Korea 2016 [Internet]. Cheongju: Korea Centers for Disease Control and Prevention, 2017 [cited 2018 Jul 3]. Available from: http://www.cdc.go.kr/CDC/info/CdcKrInfo0302.jsp?menuIds=HOMEoo1-MNU1132-MNU1138MNUoo38\&fid=32\&q_type=\&q_value $=\&$ cid=73878\&page Num $=$.

14. Kim DH, Kim HJ, Park SK, et al. Treatment outcomes and long-term survival in patients with extensively drug-resistant tuberculosis. Am J Respir Crit Care Med 2008;178:10751082.

15. Kwak N, Kim HR, Yoo CG, Kim YW, Han SK, Yim JJ. Changes in treatment outcomes of multidrug-resistant tuberculosis. Int J Tuberc Lung Dis 2015;19:525-530.

16. Jeong BH, Jeon K, Park HY, et al. Outcomes of pulmonary MDR-TB: impacts of fluoroquinolone resistance and linezolid treatment. J Antimicrob Chemother 2015;70:31273133 .

17. Kang YA, Shim TS, Koh WJ, et al. Choice between Levofloxacin and moxifloxacin and multidrug-resistant tuberculosis treatment outcomes. Ann Am Thorac Soc 2016;13:364-370.

18. Ho J, Jelfs P, Sintchenko V. Fluoroquinolone resistance in non-multidrug-resistant tuberculosis-a surveillance study in New South Wales, Australia, and a review of global resistance rates. Int J Infect Dis 2014;26:149-153.

19. Gunther G, van Leth F, Alexandru S, et al. Multidrug-resistant tuberculosis in Europe, 2010-2011. Emerg Infect Dis 2015;21:409-416.

20. Chien JY, Lai CC, Tan CK, Yu CJ, Hsueh PR. Direct observation therapy-plus can prevent acquired resistance to fluoroquinolones among patients with multidrug-resistant tuberculosis in Taiwan. Clin Infect Dis 2013;56:1054- 
1055 .

21. Park IN, Hong SB, Oh YM, et al. Impact of short-term exposure to fluoroquinolones on ofloxacin resistance in HIV-negative patients with tuberculosis. Int J Tuberc Lung Dis 2007;11:319-324.

22. Huang TS, Kunin CM, Lee SSJ, Chen YS, Tu HZ, Liu YC. Trends in fluoroquinolone resistance of Mycobacterium tuberculosis complex in a Taiwanese medical centre: 1995-2003. J Antimicrob Chemother 2005;56:1058-1062.

23. Kim CK, Shin SY, Kim HJ, Lee K. Drug resistance patterns of multidrug- and extensively drug-resistant tuberculosis in Korea: amplification of resistance to oral second-line drugs. Ann Lab Med 2017;37:323-326.

24. Long R, Chong H, Hoeppner V, et al. Empirical treatment of community-acquired pneumonia and the development of fluoroquinolone-resistant tuberculosis. Clin Infect Dis 2009;48:1354-1360.

25. Devasia RA, Blackman A, Gebretsadik T, et al. Fluoroquinolone resistance in Mycobacterium tuberculosis: the effect of duration and timing of fluoroquinolone exposure. Am J Respir Crit Care Med 2009;180:365-370.
26. Migliori GB, Langendam MW, D'Ambrosio L, et al. Protecting the tuberculosis drug pipeline: stating the case for the rational use of fluoroquinolones. Eur Respir J 2012;40:814-822.

27. Selvakumar N, Kumar V, Balaji S, et al. High rates of ofloxacin resistance in Mycobacterium tuberculosis among both new and previously treated patients in Tamil Nadu, South India. PLoS One 2015;10:e0117421.

28. Jabeen K, Shakoor S, Malik F, Hasan R. Fluoroquinolone resistance in Mycobacterium tuberculosis isolates from Pakistan 2010-2014: implications for disease control. Int J Mycobacteriol 2015;4 Suppl 1:47-48.

29. Jabeen K, Shakoor S, Hasan R. Fluoroquinolone-resistant tuberculosis: implications in settings with weak healthcare systems. Int J Infect Dis 2015;32:118-123.

30. Korea Centers for Disease Control and Prevention. Annual report on the notified tuberculosis patients in Korea 2014 [Internet]. Cheongju: Korea Centers for Disease Control and Prevention, 2015 [cited 2018 Jul 3]. Available from: http://www.cdc.go.kr/CDC/cms/content/mobile/56/64356_ view.html. 